\title{
Global research trends of drug-induced liver injury (DILI) in the past two decades: a bibliometric and visualized study
}

\author{
Dan Liang ${ }^{1 \#}$, Yanping Guan ${ }^{1 \#}$, Jianhong Zhu ${ }^{1 \#}$, Junyan $\mathrm{Wu}^{1}$, Xiaoxia $\mathbf{Y u}^{1}$, Kaifeng Qiu ${ }^{1}$, Zhichao $\mathrm{He}^{1}$, \\ Qing $\mathbf{H e}^{2}$
}

${ }^{1}$ Department of Pharmacy, Sun Yat-sen Memorial Hospital, Sun Yat-sen University, Guangzhou, China; ${ }^{2}$ Department of Surgical Intensive Care Unit, Sun Yat-sen Memorial Hospital, Sun Yat-sen University, Guangzhou, China

Contributions: (I) Conception and design: D Liang, Q He; (II) Administrative support: Q He; (III) Provision of study materials or patients: D Liang; (IV) Collection and assembly of data: Y Guan, J Zhu; (V) Data analysis and interpretation: D Liang, Y Guan, J Zhu, J Wu, X Yu, K Qiu, Z He; (VI) Manuscript writing: All authors; (VII) Final approval of manuscript: All authors.

\#These authors contributed equally to this work.

Correspondence to: Qing He, MD. Department of Surgical Intensive Care Unit, Sun Yat-sen Memorial Hospital, Sun Yat-sen University, Guangzhou 510120, China. Email: heqing@mail.sysu.edu.cn.

\begin{abstract}
Background: Drug-induced liver injury (DILI) has become one of the most common and serious adverse drug reactions owing to its high morbidity and leading cause of hepatic failures, which had attracted great attentions worldwide in the past two decades. A need therefore exists to conduct a bibliometric and visualized analysis of the global research trends on DILI with the aim of identifying the status of current research and prioritize the future research areas.
\end{abstract}

Methods: Publications related to DILI from 1999 to 2019 were extracted through an electronic search of the Web of Science (WoS) and PubMed databases. VOSviewer software was used to perform visualized analysis of bibliographic coupling, co-authorship, co-citation and co-occurrence, and to assess the global trends.

Results: Totally of 6,563 publications were identified based on the inclusion criteria. It is noted that the global trends of literatures on DILI showed a significant increase over time and more importantly predict the relevant publications will rise continuously in the coming decades. The United States led in terms of publication numbers with the most citations (the highest $\mathrm{H}$-index). Hepatology made great contributions to the highest H-index and the most citations of publications. University of North Carolina was regarded as the most contributive institution. Notably, after conducting co-occurrence and cluster analysis of keywords and citation networks, it is shown that the pathogenesis, clinical manifestations, diagnosis and risk factors of this field will become the most prominent research hotspots.

Conclusions: The mapping results provides global development trends of publications on DILI from 1999 to 2019. Future studies focused on the diagnosis and predisposing factors of this field will be encouraged.

Keywords! Drug-induced liver injury (DILI); global trends; bibliometric analysis; visualized analysis

Submitted Apr 23, 2021. Accepted for publication Jul 13, 2021.

doi: $10.21037 /$ apm-21-981

View this article at: https://dx.doi.org/10.21037/apm-21-981

\section{Introduction}

Drug-induced liver injury (DILI), a type of liver injury, maybe induced by various prescription or over-the-counter chemical drugs (active ingredients or metabolites), biological preparations, traditional Chinese medicine, natural medicine, healthcare products, dietary supplements and even the excipients in pharmaceutical formulations (1-4). In clinical practice, it is hard to analyze the absolute incidence of DILI accurately for the reason that symptoms of DILI may vary, ranging from serious hepatitis with 
jaundice to asymptomatic assessments of liver enzymes, and many uncertain factors can result to DILI (3). According to the references $(1,5,6)$, about 10 to 19 people are diagnosed with DILI per 100,000 people, $30 \%$ of whom are accompanied by jaundice. About $3 \%$ to $5 \%$ of DILI patients require hospitalization due to jaundice increasing continuously $(6,7)$. Drug-induced acute liver failure is the main cause of acute liver failure in the United States, and more than $50 \%$ of DILI patients suffers from acute liver failure $(6,8)$.

Given the majority of relevant literatures, hundreds of active ingredients are confirmed or estimated to be associated with DILI and sometimes the degree of liver impairments may increase the risk of severe clinical outcomes, hospitalization and even death $(2,9,10)$. DILI refers to a variety of events related to multiple mechanisms, such as active metabolites formation, inhibition of hepatic transporters, drug-induced mitochondrial dysfunction, immune system dysfunction, environmental and genetic/ non-genetic factors (11). Currently, since there are no specific biomarkers or tests for DILI, its diagnosis is still difficult because the diagnosis should be made after strictly excluding other causes of liver disease, evaluating the temporary association between abnormal liver tests and usage of drugs, searching a specific clinical drug biomarker, and estimating the liver function upon drug withdrawal $(12,13)$. The paramount importance to treating suspected DILI is to pause the culprit drug promptly. There are several types of therapeutic methods for particular causes of DILI, such as corticosteroid for DILI patients with hypersensitivity or autoimmune characteristics, $\mathrm{N}$-acetylcysteine (NAC) for acetaminophen poisoning, ursodeoxycholic acid for cholestatic DILI (13).

In recent years, the field of DILI has been rapidly expanding in terms of the mechanisms of DILI, risk factors and diagnostic tools. Many large registries such as US DILI Network (DILIN), Spanish DILI registry, and Latin American DILI Network have continuously updated their data in the past years $(4,12)$. It is very significant to realize the developmental trends of DILI-related publications in different countries, institutions, authors and journals, and identify the status of current research and prioritize the future research areas. Now there are many systematic manuscripts related to DILI hotspots, such as novel drugs causing DILI, the renewed risk factors and the update of diagnostic tools, and so on. However, no such a strictly scientometric study exists to describe the current and future directions of DILI.
Bibliometric is a statistical tool that is used to quantitative evaluate the influence of research literatures within a certain time frame, research countries, institutions, journals and research collaboration for any specific topic selected for study (14). Recently, bibliometric analysis extensively used in a series of research fields (15-17). Comparing the change of research hotspots by time, it provides a simple way to predict the future direction of this research field. The Web of Science (WoS) database, the world's original index for scholarly and scientific study, is most frequently selected for bibliometric analysis for the reason that it provides a wide coverage of manuscripts with accurate and complete information.

Consequently, our study conducted a quantitative analysis of literatures related to this field during the last two decades by classifying the relevant publications at different subgroups, such as countries/regions, journals, authors, categories, research institutions, and keywords. This study attempts to depict the current state of DILI and predict the global trends of the development of research in DILI.

\section{Methods}

\section{Data sources}

In this study, the global DILI-related literatures published during the period 1999 to 2019 were mainly retrieved from the WoS core collection database and PubMed database. The publications' data were applied to bibliometric analysis. It is considering that these data were downloaded from public databases. Ethical approval has no application to the study.

\section{Search strategies}

All the articles related to DILI were screened in WoS (Clarivate analytics, https://clarivate.com/products/webof-science/) and PubMed [United States National Library of Medicine (NLM), https://pubmed.ncbi.nlm.nih.gov] databases by two researchers (D. Liang and J. Zhu), with databases expiration of 20 June 2020, and cross-validated to guarantee reproducibility of screening methods using the following search terms. The specific screening cycle were set from January 1, 1999 to December 31, 2019 in both databases. The search terms were set as follows: theme=Drug Induced Liver Injury OR Drug-Induced Liver Injury AND Publishing Year $=(1999-2019)$ AND Document Types $=($ REVIEW or ARTICLE $)$ AND 
language $=($ English $)$ AND Indexes $=$ SCI-EXPANDED, SSCI, A\&HCI, CPCI-S, CPCI-SSH, ESCI, CCREXPANDED, IC. As for the searching in PubMed database, the search terms were as follows: "Chemical Drug Induced Liver Injury"[MeSH Terms] OR “Chemical Drug Induced Liver Injury"[All Fields]) AND ((“1999/01/01”[PDAT]: “2019/12/31"[PDAT]) AND English [language]. The types of literature were included clinical study, review, case reports, and meta-analysis in PubMed database. However, after merging the datasets obtained from the two databases, case reports and book chapters were not included the final bibliometric analysis for case reports could not be selected in WoS and only few of book chapters in WoS and PubMed database.

\section{Data collection}

In collaboration with an information specialist and stakeholders in our team, the data management process was checked and confirmed independently. Support by EndNote $\mathrm{X} 8.2^{\mathrm{TM}}$ bibliographic software that allows to remove the reduplicative articles and store datasets retrieved from all articles. To identify the irrelevant studies, we conducted hand searches in the reference lists for excluding all the potentially literatures.

Two authors completed the data extraction independently and checked the article titles and abstracts, the selected articles whether if they met the search terms. To discuss or to seek assistance from outside experts were used to resolve any disagreements to achieve consensus. Finally, the data were visualized graphically by two authors with GraphPad Prism 8.

\section{Statistical analysis}

\section{Bibliometric analysis}

The bibliometric analysis is an analytical method involving statistics and mathematics. By applying the intrinsic functions of WoS, the developmental dynamics and characteristics of publications would be described based on the prior eligible publications. The publications amounts of each year, the change of citations frequency and the quality of the published articles will all be forecast in different countries/regions/constitutions. The H-index implied that a scholar, a country or an author published $\mathrm{H}$ papers each of which has been cited in other publications at least $\mathrm{H}$ times, which was regarded as a sign that how much influence they have in the scientific research (18). Therefore, the H-index reflects the scientific article impact of a scholar, a country, or an author.

\section{Visualized analysis}

VOSviewer 1.6.15 software was applied for visualizing and constructing of a visual map based on the pre-setting search terms in WoS and PubMed databases analysis. This software was used to conduct bibliographic coupling, coauthorship, and co-citation analysis the dataset collected from the eligible publications, including journals, authors, institutions, and countries. Finally, the clustering analysis of research hotspots were visualized and the developmental trends were also forecast through keyword co-occurrence networks.

\section{Results}

\section{Trends in global publications (based on WoS database)}

\section{Contributions of countries}

From 1999 to 2019, a total of 6,477 and 3,075 literatures were identified in WoS and PubMed databases, respectively, among of which about 6,563 articles met the criterions were recruited for bibliometric analysis. In WoS database, the distribution of publications related DILI in different countries is shown in Figure 1A. The United States is the leading country for it has published the largest numbers of articles in this field (1,917, accounting for $29.21 \%$ ), followed by China (1,242, accounting for $18.92 \%)$, Japan (584 papers, accounting for $8.90 \%$ ), India (386 papers, accounting for $5.88 \%$ ) and United Kingdom (378 articles, accounting for $5.76 \%$ ). In summary, publications in the top 5 countries $(68.7 \%)$ paly a much larger role than that in the rest of other countries.

\section{Amount of global publications per year}

The number of publications on DILI exhibited a positive growth trend from 2009 to 2019 (shown in Figure 1B). Globally, publications had the maximum number in 2019 with a total of 823 articles, while only 58 related articles published in 2000. The fluctuations of annual citations were showed in Figure $1 C$. The citations per year of the documents showed a rapid growth from 1999 to 2016, but there was a decline from 2017 to 2019, with a peak in 2016 $[13,444]$ and a tough in 2019 [6,382].

\section{Predicted trends in global publication}

The time series model, a common regression analysis, was used to describe the time curve of number of articles from 
A
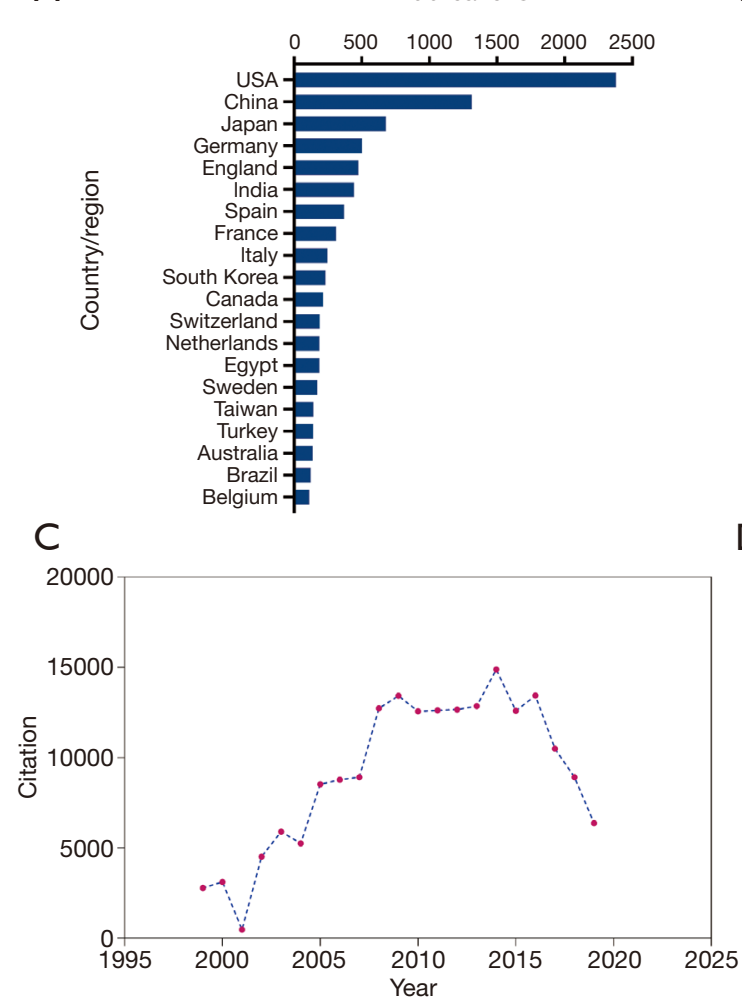

B

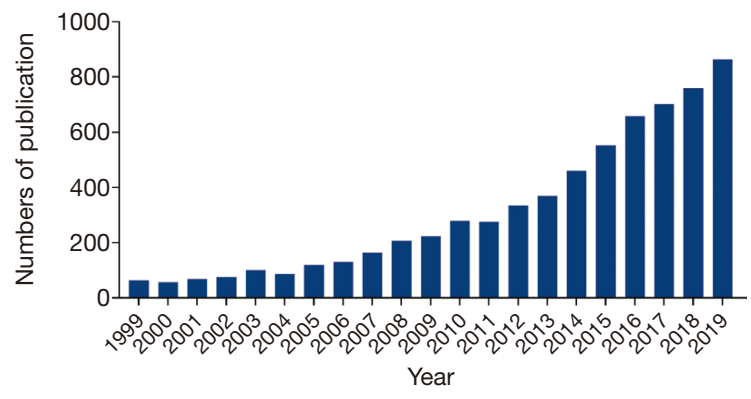

D

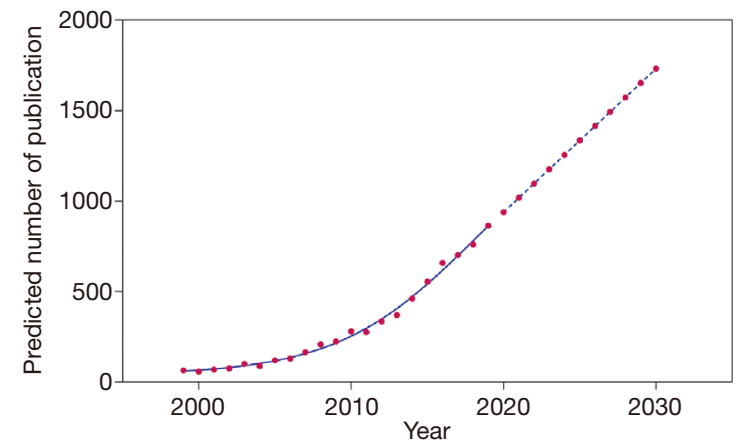

Figure 1 Global trends and countries contributing to drug-induced liver injury research based on the WoS database. (A) The sum of relevant publications from the top 20 countries. (B) The number of publications per year during the last two decades. (C) The number of citations per year during the last two decades (D). Model fitting curves of growth trends in worldwide publications and prediction of future publication numbers.

which the trend number of global publications is possible to be predicted (Figure 1D). The time curve shows that the global publishing outputs on DILI is currently at a rapidly growing stage, but it will be expected to keep with a steadily increasing rate in the next ten years.

\section{Distribution of publication quality by country (based on WoS database)}

\section{Total citation frequency}

It was found that publications related to DILI from U.S. had the highest total citation frequency (76,176 times). China ranked second in total citation frequencies (18,593 times), followed by England (15,163 times), Japan (11,153 times), and Germany (10,597 times) (Figure 2A).

\section{Average citation frequency}

Switzerland ranked first in average citations (43.24), followed by England (40.11), USA (39.74), Sweden (39.41) and France (39.25) (shown in Figure 2B).

\section{H-index}

The publications from USA had the highest H-index [123], followed by England [63], China [54], Japan [50], and Germany [49] (Figure 2C).

\section{Bibliometric coupling analysis}

Bibliometric coupling measures the association among the articles cited in the same manuscript. If two publications cite the same references, they are "coupled", indicating that the two publications may share the same references and produce the knowledge domain map of countries/ regions, institutions, and journals. The strength of the link between them shows the number of references cited in the two articles, which could be extended to analyze the link 
A

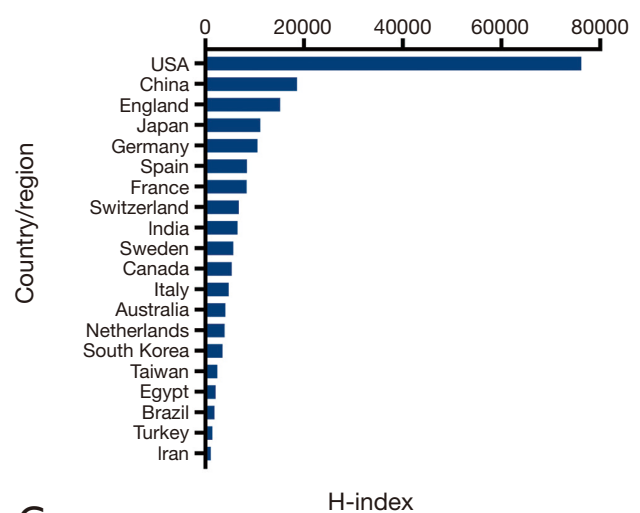

C

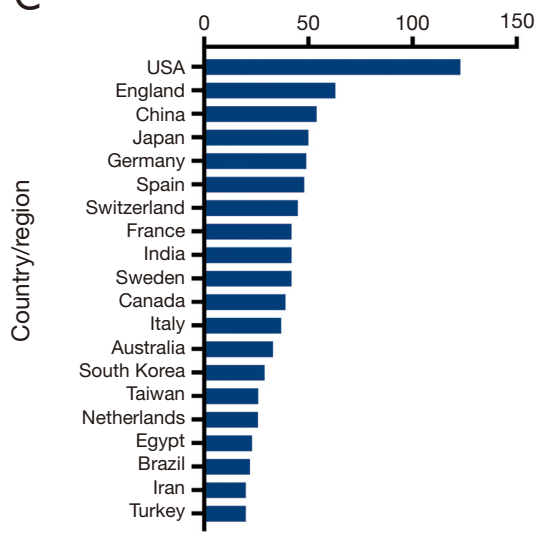

B

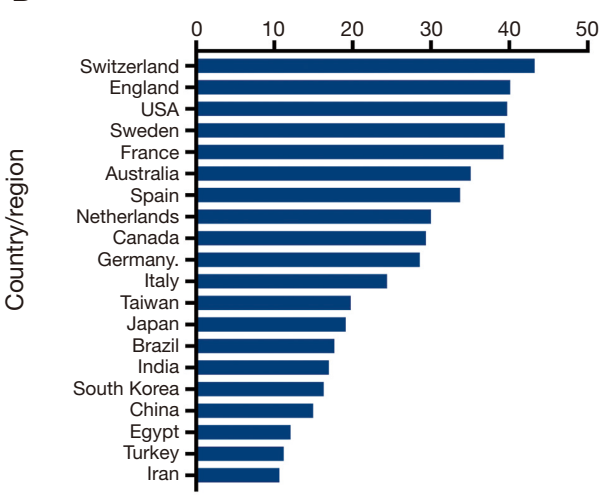

Figure 2 Citation frequency and H-index published in drug-induced liver injury in several countries. (A) Total citations of research articles from different countries. (B) The average citations per article from different countries. (C) $\mathrm{H}$-index of publications from different countries.

strength of journals, institutions, and countries publishing papers on specific topics to reflect the degree of bibliometric coupling (15).

The strength of a link between two publications indicates the number of cited references these two publications have in common. This can be scaled up to analyze the link strength of journals, institutions, and countries which have published papers within a particular subject matter, reflecting the degree of bibliographic coupling.

\section{Journals}

We used VOSviewer software to analyze the journal titles of publications in this study. Figure $3 A$ shows the total line strength of 267 identified journals. The top five journals regarding the total link strength with nodes sizes are Hepatology (total link strength $=96,294$ times), Toxicological Sciences (95,441 times), Fournal of Hepatology (68,983 times), Chemical Research in Toxicology (67,560 times) and PLoS One (45,338 times). WoS database showed the following journals got the top five rank in H-index and citations: Hepatology (2019 H-index $=63$; citations =11,624 times), Toxicological Science (45; 7,226), fournal of Hepatology (45; 5,867), Chemical Research in Toxicology $(36 ; 3,714)$ and PLoS One $(27 ; 2,254)$. Toxicological Science had the most publications ( $\mathrm{n}=150,2019$ impact factor $=3.70)$, followed by Hepatology $(123,14.68)$, PLoS One (101, 2.94), Chemical Research in Toxicology (91, 3.18), Journal of Hepatology $(82,20.58)$.

\section{Institutions}

A total of links strength of 294 institutions were analyzed by using VOSviewer (Figure 3B). The top five institutions with the large link strength were University of North Carolina (total link strength $=31,530$ times), University of Michigan (27,940 times), University of Malaga (26,399 times), University of Liverpool (22,304 times) and US Food Drug Administration (21,577 times). Table 1 showed the top 10 productive institutions based on data from WoS database. The H-index and the totalcitations of University of North Carolina, US Food Drug 

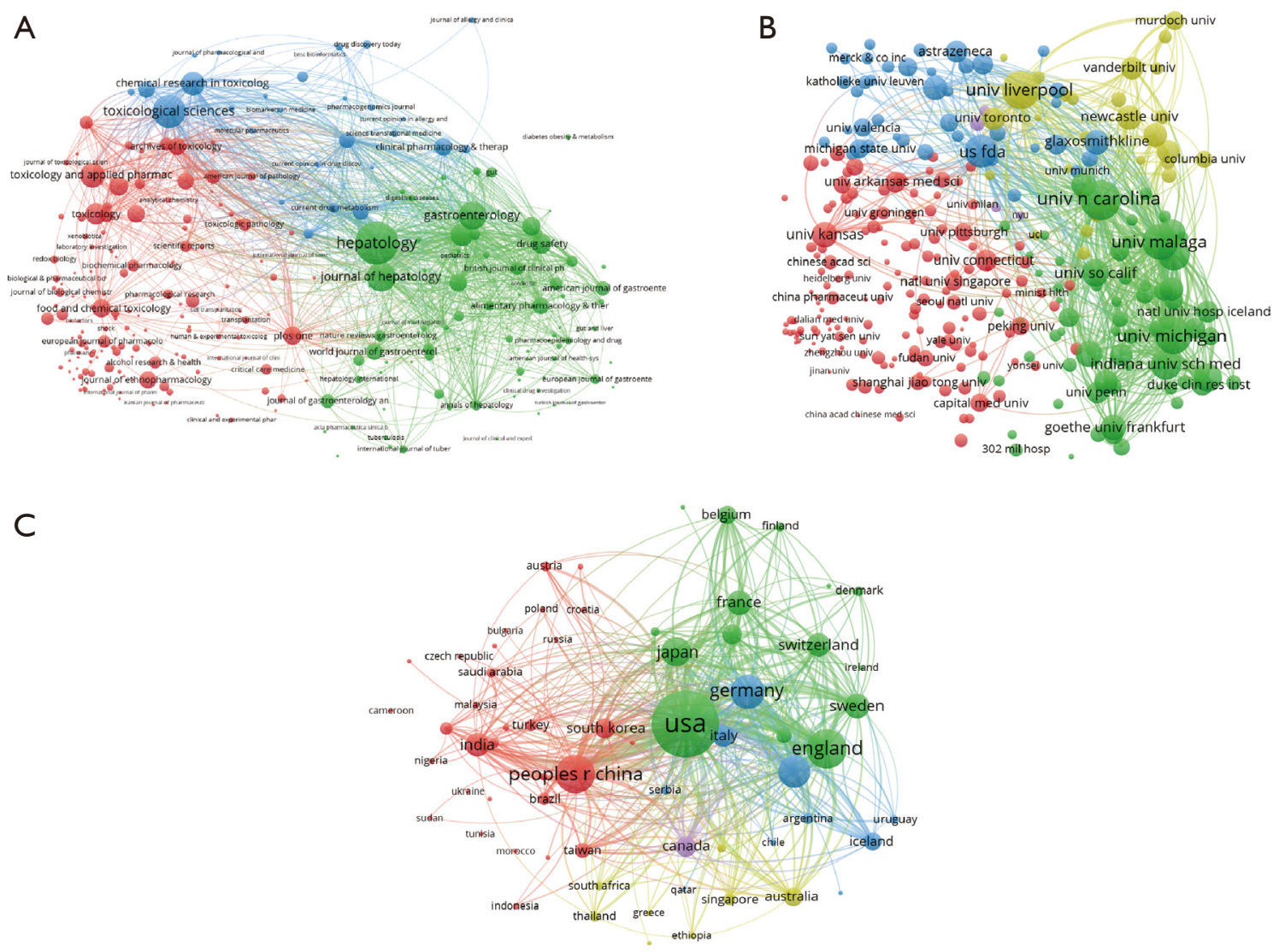

Figure 3 Bibliographic coupling analysis of global research on drug-Induced Liver Injury based on the WoS database. Mapping of (A) 267 identified journals, (B) 294 institutions, and (C) 65 countries in this research field. The line between two points in the map state that two journals/institutions/countries had established a similar relationship. The thicker line indicates, the closer connection between the two entities.

Table 1 Top 10 productive institutions on DILI from 1999 to 2019

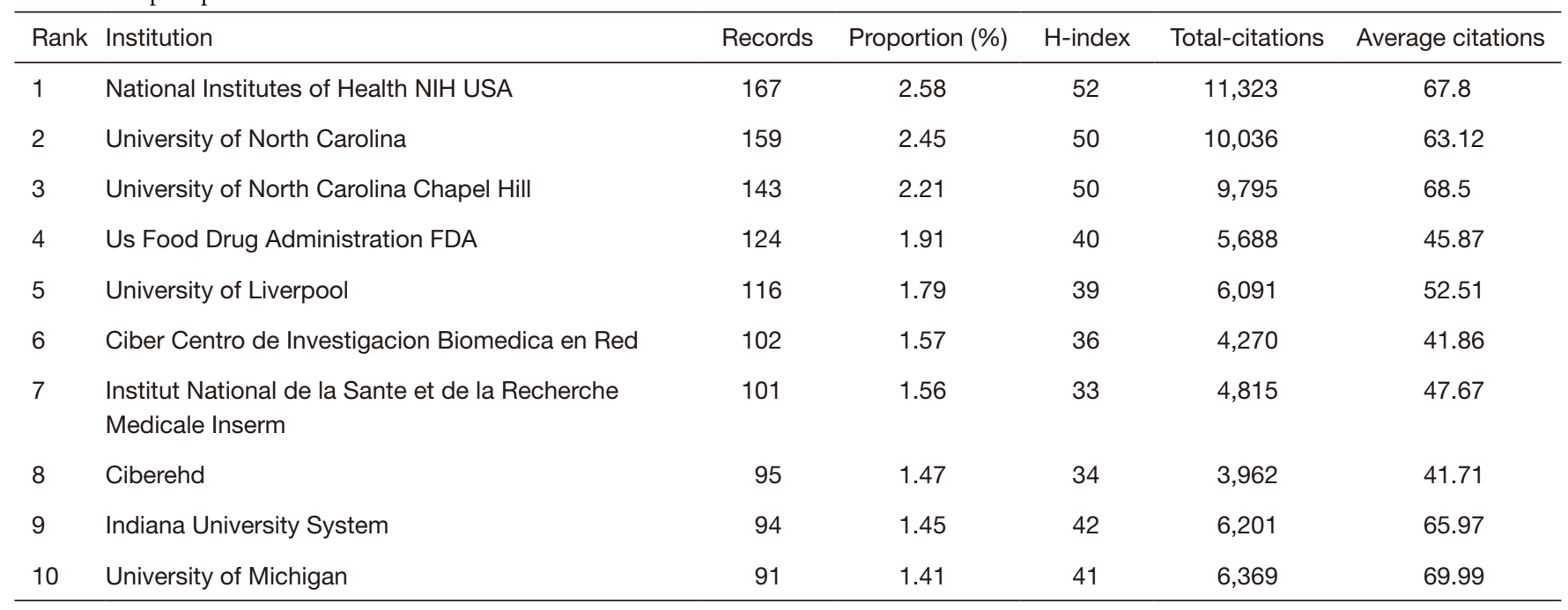

DILI, drug-induced liver injury. 
A

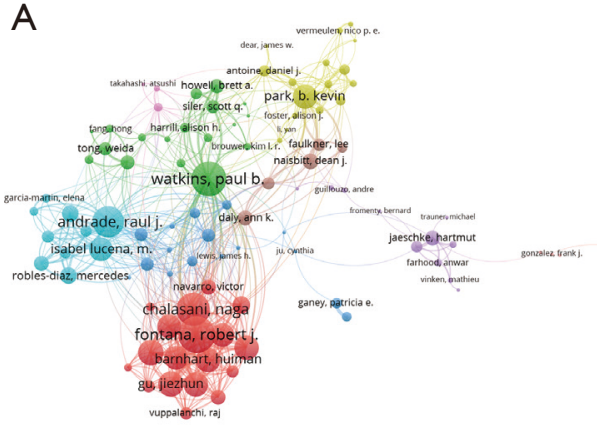

B

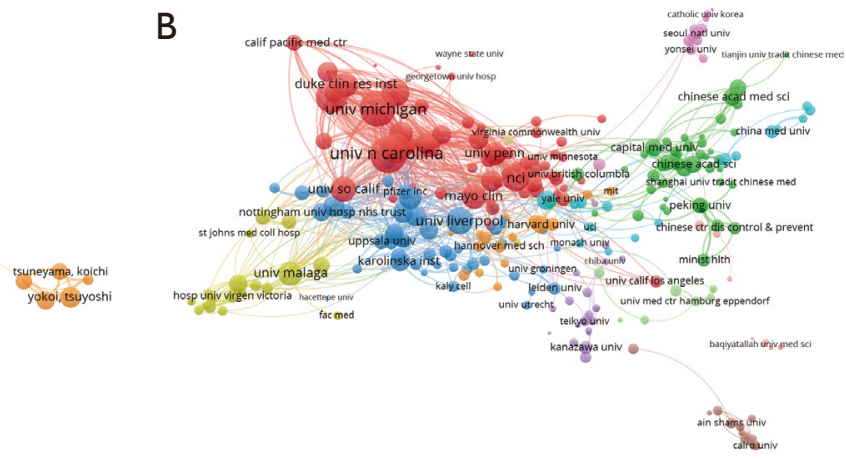

C

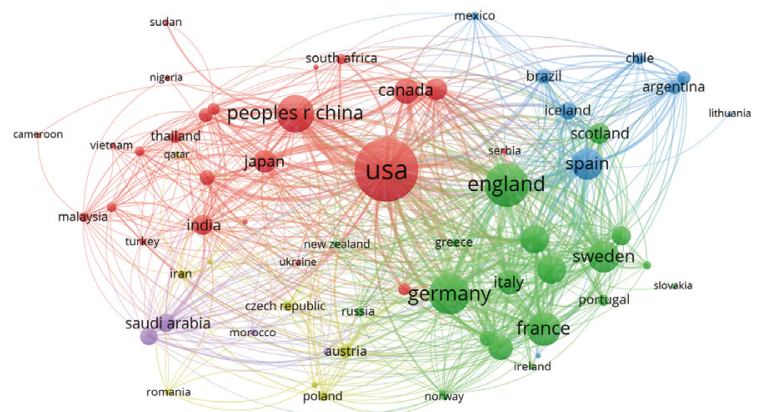

Figure 4 Co-authorship analysis of global research on drug-induced liver injury based on the WoS database. Mapping of (A) 139 authors, (B) 294 institutions, and (C) 55 countries on the research area. The co-authorship frequency is represented by the size of points. The line between two points indicates the established collaboration between two authors/institutions/countries. The thicker line states, the closer collaboration between the two entities.

Administration, University of Liverpool and University of Michigan were in the top 5 . The bibliometric coupling strength ranking of the constitutions were basically consistent with the $\mathrm{H}$-index and the-total citations ranking. Although University of Malaga isn't to maintain the previous ten were ranked, it has a large link strength.

\section{Countries}

A total of line strength for 65 countries with at least 5 articles in this field were analyzed by using VOSviewer (Figure 3C). The top five countries with the large link strength were USA (total link strength $=643,306$ times), England (310,850 times), China (293,075 times), Germany (252,385 times) and Spain (227,454 times).

\section{Co-authorship analysis}

Co-authorship network analysis is designed to discovery the connection among researchers based on their numbers of co-authored literatures and produce the knowledge domain map of relative authors, organizations and countries/regions on the purpose of describing the collaboration network relationship in DILI. The size of the circle in the network shows the collaborative degree of co-authorship. It includes the authors, organizations, countries/regions, and the total link strength of the connecting line represents the strength of the cooperation. Indeed, description of the collaboration network among the creative authors, organizations and countries/regions is crucial and can offer worthy information for those independent investigators to seek cooperation, for institutions and countries/regions to develop regional cooperation and facilitate the academic exchange.

\section{Authors}

A total of 139 authors, each of whom have published over 10 relative articles, were analyzed by using VOSviewer (Figure $4 A$ ). These authors were identified from all publications retrieved in this study. The top five authors regarding the total link strength are Fontana $\mathrm{R}$ (total link strength $=229$ times), Watkins $\mathrm{P}$ (211 times), Chalasani $\mathrm{N}$ (197 times), Andrade R (179 times) and Gu J (130 times).

\section{Institutions}

A total of 294 institutions with the minimum number of 
publications more than 10 in this area were analyzed to construct the co-authorship network by using VOSviewer (Figure $4 B$ ). The top five universities were University of North Carolina (total link strength $=386$ times), University of Michigan (361 times), National Institute \& Diabetes and Digestive and kidney Disease of the National Institutes of Health (268 times), Duke University (234 times), Indian University School of Medicine (207 times).

\section{Countries}

Fifty-five countries and regions this field were evaluated with VOSviewer (Figure 4C). The top five countries with largest DILI based on publications with the minimum number of articles of a country over 5 were USA (969 times), England (492 times), Germany (440 times), China (366 times) and France (352 times).

\section{Co-citation analysis}

Co-citation analysis investigates the association between two items when they cited in a same document. In a cocitation map of publications, the relatedness of items cited several times by different researchers can be observed conveniently and efficiently from a mass of references, providing adequate information for author in the same research field. It is the same that the size of the circle in the network presents the degree of co-citation including the authors, references, journals, and the total link strength of the connecting line represents the strength of the cooperations.

\section{Authors}

A total of 1,166 authors with more than 30 articles in this area were analyzed by using VOSviewer (Figure $5 A$ ).

The top five authors regarding the total link strength were Teschke, R (total link strength $=34,748$ times), Jaeschke H (31,834 times), Andrade RE (29,736 times), Bjornsson E (28,722 times) and Lucena MI (24,582 times).

\section{References}

Co-cited references can show the frequency of publications cited together, therefore they can be viewed as a knowledge base in a particular field (19). Articles with the minimum number of citations of a cited reference over 20 were analyzed by using VOSviewer and 1,121 references were exhibited in Figure $5 B$. The top five references with the total link strength were Andrade RJ, 2005, Gastroenterology (total link strength =8,917 times); Daly AK, 2009, Nat Genet (7,991 times);
Danan G, 1993, 7 Clean Epidemiol (7,487 times); Ostapowicz G, 2002, Ann Intern Med (7,417 times) and Chalasani N, 2008, Gastroenterology (7,165 times).

\section{Journals}

The journal names of co-citation analysis with all the publications were analyzed by using VOSviewer software. A total of 1,296 journals with more than 30 co-citations (Figure $5 C$ ) in this field were analyzed. The top five references with large total link strength were Hepatology (total link strength $=1,168,276$ times); Gastroenterology (581,483 times); Fournal of Hepatology (521,586 times); Fournal of Biological Chemistry (433,541 times); Drug Metabolism and Disposition (385,385 times).

\section{Co-occurrence terms and cluster analysis}

Co-occurrence analysis investigates the co-occurrence links between research terms. The link strength of cooccurrence illustrated the number of documents where two topics appear at the same time. Co-occurrence analysis can reveal up-to-date knowledge of the current status of research topics in this field. In addition, it can be proved to be very useful to explore the hotspots and future directions in research related DILI. Also, it helps to monitor the development of academic programs.

The network visualization term map for DILI research conducted globally from 1999 to 2019 (Figure 6). A total of 19,326 terms have been identified through the selected publications, but only 545 of terms repeated more than 20 times. In the term map (Figure 6A), it is noted that four thematic research clusters or regions were classified by 545 co-occurring terms with four different colors. The terms with similar research topics were combined together under a same catalog, and the 4 main clusters were pathogenesis, diagnosis/risk factors/treatment, clinical manifestations and pathology of DILI. For the pathogenesis cluster (in red color), the primary keywords were "oxidative stress", "mice", "inflammation", "gene-expression”, "expression”, "celldeath" and "mitochondria". For the diagnosis/risk factors/ treatment cluster (in green color), the main keywords were "drug-induced liver injury", "causality assessment", "diagnosis", "risk-factor", "herbal hepatotoxicity" and "transplantation". For clinical manifestation cluster, the primary keywords were "cholestasis", "injury", "salt export pump", "liver fibrosis", "damage". As for the pathology Cluster, "antioxidant", "lipid-peroxidation" and "rats" were served as main keywords. 

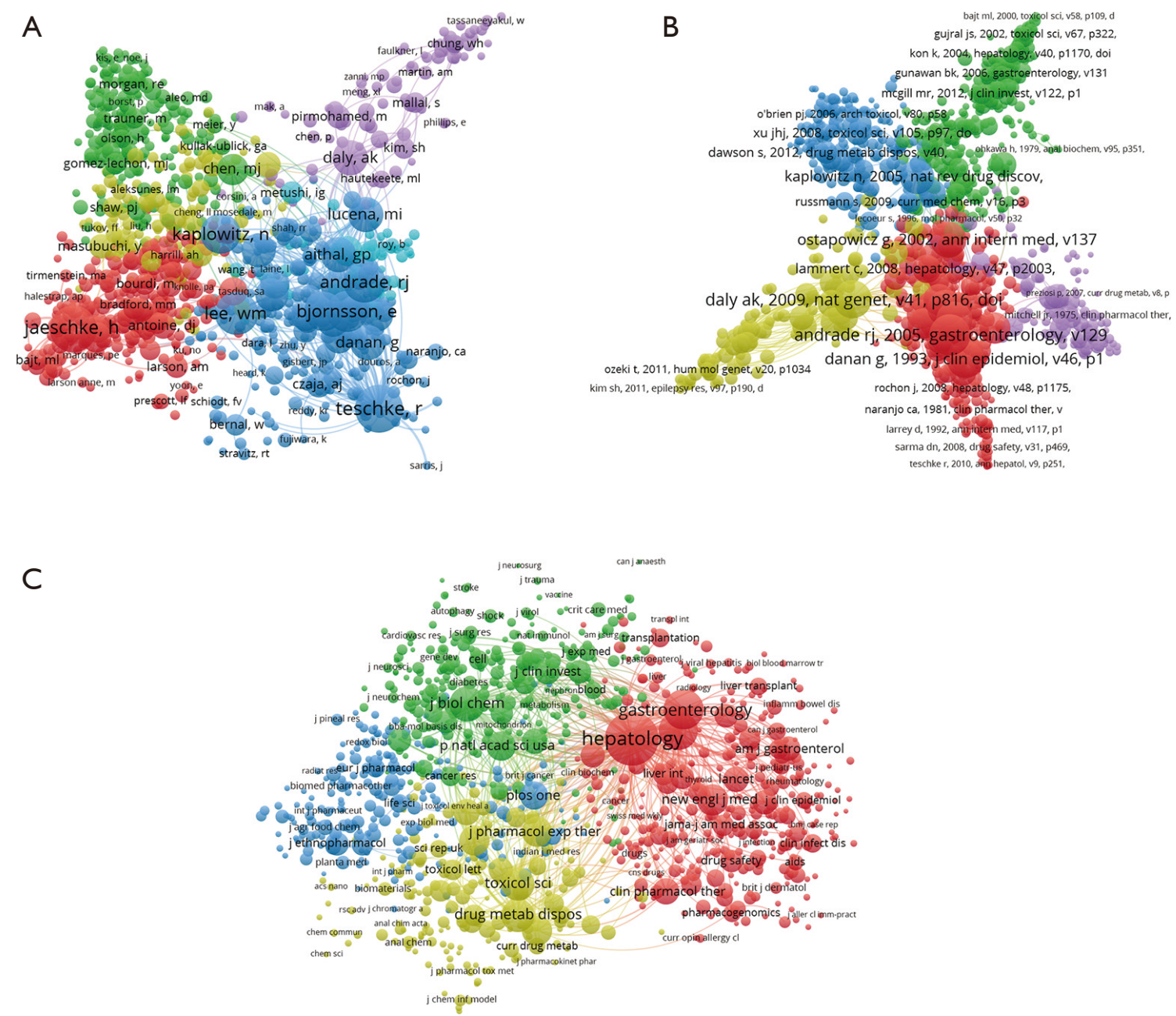

Figure 5 Co-citation analysis of global research on drug-induced liver injury based on the WoS database. The same color point represents the same research topic. (A) Mapping of co-cited authors in the field. Points marked by different colors indicates the 1,166 cited authors. (B) Mapping of co-cited references in the field. Points marked by different colors indicates the 1,121 cited references. (C) Mapping of co-cited journals in the field. Points marked by different colors indicates 1,296 identified journals. The citation frequency is represented by the size of the points. A line between two points suggests both cited in the same paper or journal. The shorter the line, the closer the link between two entities.

Density visualization map (Figure 6B) was exported by VOSviewer. The times of occurrence of a key word were defined as the color of the area. The larger the times, the warmer the color is. Blue corresponds with the lowest item density and red corresponds with the highest item density. VOSviewer software was used to code the color of terms according to the average time they appeared in all the included publications. The keywords with an average earlier time period were represented by the purple color, and the yellow colored keywords with an average terminal time period appeared later. Before 2012, majority of studies focused on the topics of "mechanism research" and "oxidative stress", while the latest trends show that "diagnosis" and "risk factors" might be concerned in the future.

Meanwhile, the VOSviewer software was used to identify the density based on the frequencies of the keywords which could be exhibited by the density map (Figure 6C). The higher frequencies, the more warmer color is, representing the higher density is. The research hotspots in the field were biased towards areas with larger gray values. 

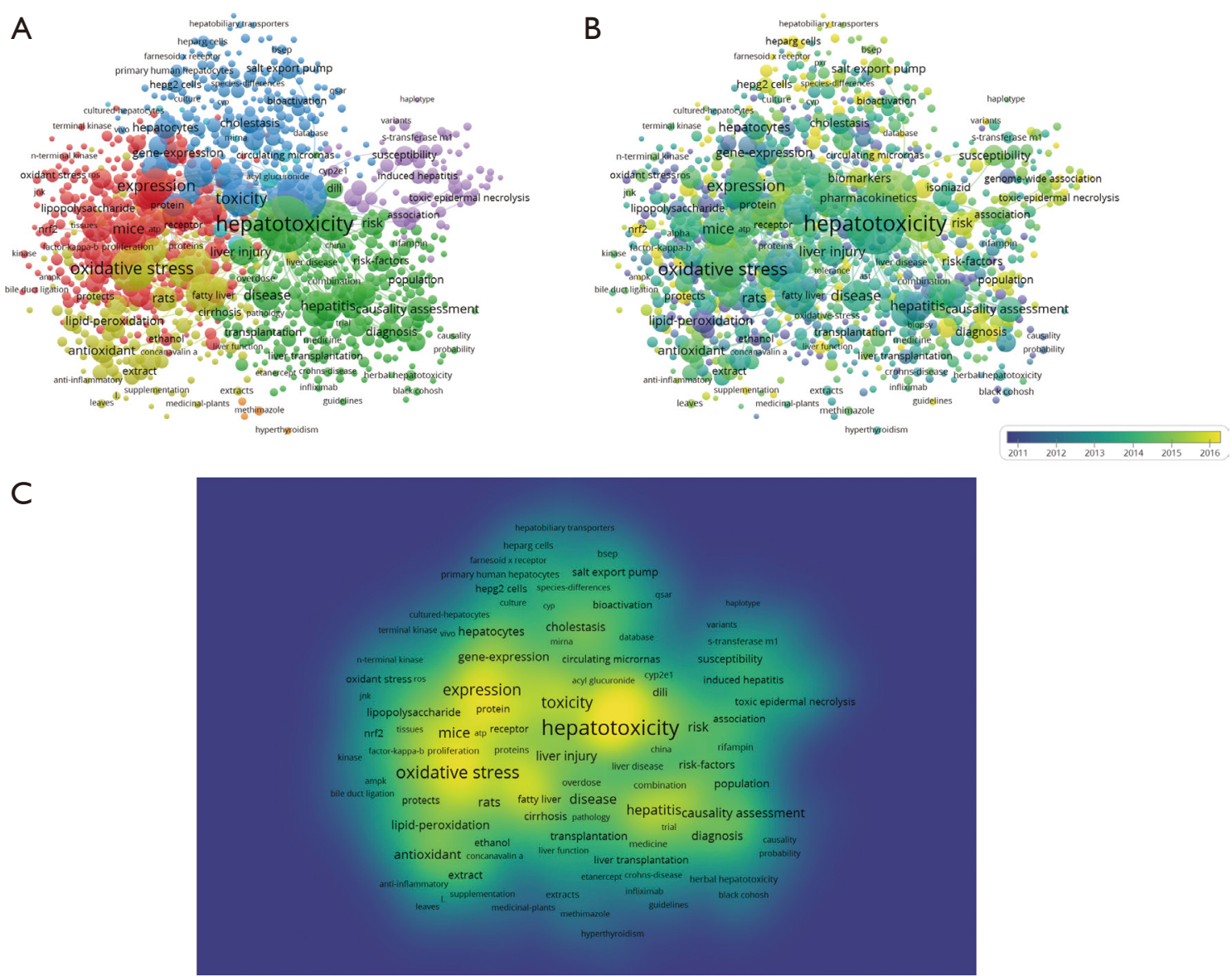

Figure 6 Co-occurrence analysis of global research on drug induced liver injury based on the WoS database. (A) Mapping of keywords in the research field. The frequency of appearance is represented by the size of the points, and the keywords are divided into four clusters: mechanistic studies (red), diagnosis studies (green), expression studies (blue), and in-vitro studies (yellow). (B) Distribution of keywords regarding the chronological order of appearance. Keywords in purple appeared the first, green the second, and yellow the latest. (C) Distribution of keywords regarding the average frequency of appearance. Keywords in yellow represented the highest frequency, followed by green, and purple.

\section{Discussion}

\section{Trends in the research field}

During the current information exploration, investigators often focus on how to conduct knowledge management and acquirement on a certain field. In our present study, we used a combination of bibliometric and visualized analysis to describe the current status in the research field on DILI. We evaluated the relatedness of individual authors, journals, organizations, countries/regions in this fast-growing research field, and the existing hotspots from 1999 to 2019, as well as the predicted concern topics in the future. To our knowledge, this is the first study to use bibliometric and visualized analysis to evaluate the knowledge map of the developmental tendency, prospective progress, and existing/ evolving hotspots that arouse the interests of researchers in the coming years.

Regarding the numbers of publications in this field, it is easily found that the developmental trends increased from 1999 to 2019, particularly with an obvious and exponential growth throughout the past ten years. Since the risk factors associated with increased susceptibility to DILI and precise diagnostic tests for IDLI are still missing, the clinical study related to DILI had attracted public's attentions during the past years (20-22). Our study shows that the publications of 294 institutions from 65 countries all over the world have 
significant bibliographic coupling among more than 6,000 publications since 1999. These figures were organized jointly to evaluate the forepassed, existing and prospective research hot topic in this certain research field. Cooccurrence terms provide a reliable reference to researchers that the research fields of "diagnosis" and "risk factors" would be appreciated in the future.

\section{Quality of global publications according to countries, institutions and journals}

\section{Countries}

The United States made great contribution in this field in terms of its total numbers of publications, H-index, total citation frequency, the top rank for bibliometric coupling and co-authorship analysis. The trends in Figure 2A,C show that the United States has the highest article quality and academic impact in the DILI research field, as well as the strongest partnership in the world. On the other hand, Switzerland has the highest number of average citation frequency in this research field. In addition, some other countries such as China, the United Kingdom, Japan, and Germany, have rapidly increased the number, quality, influence, and cooperation of publications in this field in the past two decades. In the past ten years, China has ranked $2^{\text {nd }}, 2^{\text {nd }}$, and $3^{\text {rd }}$ separately in the world in the number of publications, total citation frequency and $\mathrm{H}$-index in this field. This suggests the publications of China in this field focus not only on quantity but also on academic impact and quality.

\section{Institutions}

In our study, the link strengths of 294 institutions determined by bibliometric coupling and co-authorship analysis show the relative contribution of specific institutions to the DILI research field.

Bibliometric coupling and co-authorship analysis show that the top 5 universities may be the most powerful universities to this field in the world. The institutions that contribute the most in this field were also from the country with the highest impact in this field-the United States. Moreover, within the top five rankings of the Bibliometric coupling and co-author analysis of the institution, the University of North Carolina and the University of Michigan are ranked $1^{\text {st }}$ and $2^{\text {nd }}$, which are stronger than University of Liverpool and US Food Drug Administration, indicating that these two universities made great contribution in this field. An article of the
University of North Carolina named of "Causes, Clinical Features, and Outcomes from a Prospective Study of Druginduced Liver Injury in the United States (23)", published in 2008 , served as the most highly cited publication in this field. This article is cited by "Drug-induced liver injury: Asia Pacific Association of Study of Liver consensus guidelines" and "Consensus guidelines: best practices for detection, assessment and management of suspected acute drug-induced liver injury occurring during clinical trials in adults with chronic cholestatic liver disease" $(24,25)$. They concluded that "DILI is caused by a wide array of medications, herbal supplements, and dietary supplements".

\section{Journals}

Popular and core journals in a field are easy to be searched and they can provide many valuable documents. The link strengths of journals determined by bibliometric coupling and co-citation analysis revealed the relative the degree of relevance between a specific journal and the DILI research field. Combining citations, $\mathrm{H}$-index ranking with bibliometric coupling and co-citation analysis may be more helpful for us to judge the influence of the journal in the research field. The analysis of the bibliometric reflects Hepatology is the best affection journey in this research field, but fournal of Hepatology has the highest impact factor. The articles of Hepatology such as (Bala S et al., 2012), (Reuben A et al., 2010), (Starkey Lewis PJ et al., 2011), etc. were cited actively in other studies (26-28). In recently years, journal of Hepatology's articles mainly focused on tool of identification of causative drugs, biomarkers of diagnostic and prognostic, pathophysiology, risk factors in this field, etc. $(12,29-32)$.

\section{Future outlooks}

Bibliometric analysis is a helpful tool that can exhibit the future directions of this field through co-occurrence network maps grouped by subject areas or the published dates of articles. Hematopoietic studies, oxidative stress studies, cell death and in vitro studies are regarded as comprehensive research directions in the future, each of which contains a series of important subtropics (Figure 6A,C). However, the "remote" subject areas surrounding the visual map do not yet belong to any large clusters and have not yet established strong co-occurrence links with other major themes in the field. These topic areas consist of, such as, salt export pump, extract, and covalent binding. Although they are not the main research fields, they present a long- 
term problem of a multidisciplinary approach that deserves further study.

The earlier co-occurrence analysis map (Figure 6B) was classified by different topic area according to publication date, which can show the next few years directions. There are several yellow colored themes, such as the topic of "diagnosis" and "risk" in the spectrum map, which may arouse interests in research in the future.

\section{Strengths and limitations}

Bibliometric and visualized analyses on DILI publications in the past two decades have been conducted in our study. This research described the current status of DILI research by measuring the frequency and quality of citation, bibliographic coupling, co-authorship, co-citation, and co-occurrence. This provided a large amount of information from different perspectives and exhibited the current and future research trends in this field. As far as we all known, this is the first such research in this field. Nevertheless, the accuracy of our analysis still has certain limitations. First, only English documents were included in the WoS and PubMed databases, and the omission of non-English documents may lead to biases in the research of language publication documents. Secondly, recently published research may be underestimated due to the low frequency of citations, even though these documents are published in high-quality journals. Citation indicators in research fields will become more apparent over time, for example authors, journals, institutions, and countries.

Although some studies are published in high-quality journals, there are underestimated contributions to different analyses because they are cited with very low frequency. Over time, these existing publications with a true impact will largely affect their citation indicators for journals, authors, institutions, and countries in the field much more apparently. Moreover, this study only included the WoS and PubMed databases, while papers from other databases could not be retrieved, probably leading to inadequate data collection and different results. In addition, VOSviewer software only cites abstracts instead of full text to analyze co-occurring keywords, which makes it impossible to provide sufficient details of DILI research through abstracts alone.

\section{Conclusions}

This present study showed the global trends of DILI research and present an overview of current research hotspots in the past two decades by using bibliometric and visual analysis of publications. This study indicated that the number of published articles in this field increased rapidly in the recent years. The countries of United States and China made great contribution to this field, and "Fournal of Hepatology" is the most impactive journal. Given the complexity of DILI diagnosis, DILY's research on "diagnosis", "risk factors" and "drugs" may go through with an escalating trend.

\section{Acknowledgments}

The authors would like to thank the teams of the Surgical Intensive Care Unit and Clinical Pharmacy in the Sun Yatsen Memorial Hospital, for supporting our study.

Funding: None.

\section{Footnote}

Conflicts of Interest: All authors have completed the ICMJE uniform disclosure form (available at https://dx.doi. org/10.21037/apm-21-981). The authors have no conflicts of interest to declare.

Ethical Statement: The authors are accountable for all aspects of the work in ensuring that questions related to the accuracy or integrity of any part of the work are appropriately investigated and resolved.

Open Access Statement: This is an Open Access article distributed in accordance with the Creative Commons Attribution-NonCommercial-NoDerivs 4.0 International License (CC BY-NC-ND 4.0), which permits the noncommercial replication and distribution of the article with the strict proviso that no changes or edits are made and the original work is properly cited (including links to both the formal publication through the relevant DOI and the license). See: https://creativecommons.org/licenses/by-nc-nd/4.0/.

\section{References}

1. Björnsson ES, Bergmann OM, Björnsson HK, et al. Incidence, presentation, and outcomes in patients with drug-induced liver injury in the general population of Iceland. Gastroenterology 2013;144:1419-25, 1425.e1-3; quiz e19-20.

2. Chalasani NP, Hayashi PH, Bonkovsky HL, et al. ACG 
Clinical Guideline: the diagnosis and management of idiosyncratic drug-induced liver injury. Am J Gastroenterol 2014;109:950-66; quiz 967.

3. Fisher K, Vuppalanchi R, Saxena R. Drug-Induced Liver Injury. Arch Pathol Lab Med 2015;139:876-87.

4. Real M, Barnhill MS, Higley C, et al. Drug-Induced Liver Injury: Highlights of the Recent Literature. Drug Saf 2019;42:365-87.

5. Sgro C, Clinard F, Ouazir K, et al. Incidence of druginduced hepatic injuries: a French population-based study. Hepatology 2002;36:451-5.

6. Gabbi C, Bertolotti M. Drug-Induced Liver Injury - Types and Phenotypes. N Engl J Med 2019;381:1395-6.

7. Vuppalanchi R, Liangpunsakul S, Chalasani N. Etiology of new-onset jaundice: how often is it caused by idiosyncratic drug-induced liver injury in the United States? Am J Gastroenterol 2007;102:558-62; quiz 693.

8. Stravitz RT, Lee WM. Acute liver failure. Lancet 2019;394:869-81.

9. Andrade RJ, Medina-Caliz I, Gonzalez-Jimenez A, et al. Hepatic Damage by Natural Remedies. Semin Liver Dis 2018;38:21-40.

10. Navarro VJ, Lucena MI. Hepatotoxicity induced by herbal and dietary supplements. Semin Liver Dis 2014;34:172-93.

11. Norman BH. Drug Induced Liver Injury (DILI). Mechanisms and Medicinal Chemistry Avoidance/ Mitigation Strategies. J Med Chem 2020;63:11397-419.

12. Stephens C, Robles-Diaz M, Medina-Caliz I, et al. Comprehensive analysis and insights gained from longterm experience of the Spanish DILI Registry. J Hepatol 2021;75:86-97.

13. Katarey D, Verma S. Drug-induced liver injury. Clin Med (Lond) 2016;16:s104-9.

14. Liang YD, Li Y, Zhao J, et al. Study of acupuncture for low back pain in recent 20 years: a bibliometric analysis via CiteSpace. J Pain Res 2017;10:951-64.

15. Zhu Y, Li JJ, Reng J, et al. Global trends of Pseudomonas aeruginosa biofilm research in the past two decades: A bibliometric study. Microbiologyopen 2020;9:1102-12.

16. Zyoud SH. Global scientific trends on aflatoxin research during 1998-2017: a bibliometric and visualized study. J Occup Med Toxicol 2019;14:27.

17. Sweileh WM, Sawalha AF, Al-Jabi S, et al. Bibliometric analysis of literature on antifungal triazole resistance: 1980 - 2015. Germs 2017;7:19-27.

18. Sharma B, Boet S, Grantcharov T, et al. The h-index outperforms other bibliometrics in the assessment of research performance in general surgery: a province-wide study. Surgery 2013;153:493-501.

19. Shi J, Gao Y, Ming L, et al. A bibliometric analysis of global research output on network meta-analysis. BMC Med Inform Decis Mak 2021;21:144.

20. Andrade RJ, Chalasani N, Björnsson ES, et al. Druginduced liver injury. Nat Rev Dis Primers 2019;5:58.

21. Shen T, Liu Y, Shang J, et al. Incidence and Etiology of Drug-Induced Liver Injury in Mainland China. Gastroenterology 2019;156:2230-2241.e11.

22. Hassan A, Fontana RJ. The diagnosis and management of idiosyncratic drug-induced liver injury. Liver Int 2019;39:31-41.

23. Chalasani N, Fontana RJ, Bonkovsky HL, et al. Causes, clinical features, and outcomes from a prospective study of drug-induced liver injury in the United States. Gastroenterology 2008;135:1924-34, 1934.e1-4.

24. Devarbhavi H, Aithal G, Treeprasertsuk S, et al. Drug-induced liver injury: Asia Pacific Association of Study of Liver consensus guidelines. Hepatol Int 2021;15:258-82.

25. Palmer M, Regev A, Lindor K, et al. Consensus guidelines: best practices for detection, assessment and management of suspected acute drug-induced liver injury occurring during clinical trials in adults with chronic cholestatic liver disease. Aliment Pharmacol Ther 2020;51:90-109.

26. Bala S, Petrasek J, Mundkur S, et al. Circulating microRNAs in exosomes indicate hepatocyte injury and inflammation in alcoholic, drug-induced, and inflammatory liver diseases. Hepatology 2012;56:1946-57.

27. Reuben A, Koch DG, Lee WM, et al. Drug-induced acute liver failure: results of a U.S. multicenter, prospective study. Hepatology 2010;52:2065-76.

28. Starkey Lewis PJ, Dear J, Platt V, et al. Circulating microRNAs as potential markers of human drug-induced liver injury. Hepatology 2011;54:1767-76.

29. European Association for the Study of the Liver. Electronic address: easloffice@easloffice.eu; Clinical Practice Guideline Panel: Chair:; Panel members, et al. EASL Clinical Practice Guidelines: Drug-induced liver injury. J Hepatol 2019;70:1222-61.

30. Ashby K, Zhuang W, González-Jimenez A, et al. Elevated bilirubin, alkaline phosphatase at onset, and drug metabolism are associated with prolonged recovery from DILI. J Hepatol 2021;75:333-41.

31. Kaliyaperumal K, Grove JI, Delahay RM, et al. Pharmacogenomics of drug-induced liver injury (DILI): 
Molecular biology to clinical applications. J Hepatol 2018;69:948-57.

32. Urban TJ, Nicoletti P, Chalasani N, et al. Minocycline

Cite this article as: Liang D, Guan Y, Zhu J, Wu J, Yu X, Qiu K, He Z, He Q. Global research trends of drug-induced liver injury (DILI) in the past two decades: a bibliometric and visualized study. Ann Palliat Med 2021;10(8):8651-8664. doi: 10.21037/apm-21-981 hepatotoxicity: Clinical characterization and identification of HLA-B*35:02 as a risk factor. J Hepatol 2017;67:137-44. 